\title{
Electromagnetic Radiation in a Uniformly Moving, Homogeneous Medium
}

\author{
Johannsen, Günther
}

Published in:

Journal of Mathematical Physics

Link to article, DOI:

$10.1063 / 1.1665854$

Publication date:

1972

Document Version

Publisher's PDF, also known as Version of record

Link back to DTU Orbit

Citation (APA):

Johannsen, G. (1972). Electromagnetic Radiation in a Uniformly Moving, Homogeneous Medium. Journal of Mathematical Physics, 13(1), 78-83. https://doi.org/10.1063/1.1665854

\section{General rights}

Copyright and moral rights for the publications made accessible in the public portal are retained by the authors and/or other copyright owners and it is a condition of accessing publications that users recognise and abide by the legal requirements associated with these rights.

- Users may download and print one copy of any publication from the public portal for the purpose of private study or research.

- You may not further distribute the material or use it for any profit-making activity or commercial gain

- You may freely distribute the URL identifying the publication in the public portal

If you believe that this document breaches copyright please contact us providing details, and we will remove access to the work immediately and investigate your claim. 
$\psi_{\omega(\mathbf{k})}^{(+)}=\left[\begin{array}{c}\frac{f^{*}(\mathbf{k})}{D_{+}(\omega(\mathbf{k}))} \\ \delta(\mathbf{q}-\mathbf{k})+\frac{f^{*}(\mathbf{k}) f(\mathbf{q})}{D_{+}(\omega(\mathbf{k})[\omega(\mathbf{k})-\omega(\mathbf{q})+i \epsilon]}\end{array}\right]$

with

$D(z)=[z-M]\left(1+\int \frac{|f(\mathbf{k})|^{2}}{[M-\omega(\mathbf{k})][z-\omega(\mathbf{k})]}\right)$,

where $M$ is the physical mass of the stable $V$ particle. The $S$ matrix determined by taking the inner product of $\psi_{\omega(\mathbf{k})}^{(+)}$with the corresponding ingoing wave state of energy $\omega(\mathbf{k})$ is

$$
\begin{aligned}
S(k) & =\langle\text { in, } \mathbf{k}| \text { out }, \mathbf{k}\rangle \\
& =D_{-}(\omega(\mathbf{k})) / D_{+}(\omega(\mathbf{k})) .
\end{aligned}
$$

Briefly then, we now take nonrelativistic kinematics, $\omega(\mathbf{k})=\mathbf{k}^{2}$, and choose a particular $f(\mathbf{k}):$

$$
|f(\mathbf{k})|^{2}=\frac{2 \lambda}{\pi}[M-\omega(\mathbf{k})]\left(\frac{b^{2}}{1+k^{2} b^{2}}\right)^{2} .
$$

Thus $D(z), S(k)$, and the continuum components of $\psi_{\omega(k)}^{(+)}$ are essentially the same as the corresponding quantities in first example we considered in the pairwise theory. Thus, the Fourier transform of $\psi_{\omega(\mathrm{k})}^{(+)}$into coordinate space becomes non $-L^{2}$ as $k b \stackrel{\omega}{\rightarrow} i$, in the same way as in the previous example.

\section{CONCLUSIONS}

The analysis presented in this paper concerning the singularities of the $S$ matrix demonstrates that the non $-L^{2}$ character of the poles of $\hat{f}_{-}(k, 0)$ persists in domains closer to that of an interacting quantum field theory. From the scalar and vector coupling models it is seen that relativization does not destroy the non$L^{2}$ poles of the exponential potential. For the pair theory, classical and quantized, and for the Lee model it is now possible to make the definite statement that poles of the proper Jost function $\bar{f}_{-}(k, 0)$ correspond to non $-L^{2}$ class solutions of the associated dynamical field equation in coordinate space and that zeros of $\bar{f}_{+}(k, 0)$ to the $L^{2}$ class of solutions. A corollary of these conclusions is that non- $L^{2}$ states, as "shadow" states, possess inherently a very elegant reason for not appearing in the unitarity relation, and may be called "shadow" fields in complete correspondence with its nonrelativistic counterpart.
1 C. A. Nelson, A. K. Rajagopal, and C. S. Shastry, J. Math. Phys. 12, 737 (1971). The present paper is its sequel.

2 S. T. Ma, Phys. Rev. 69,668 (1946); 71, 195 (1947). See Ref. 1, where more recent work on such singularities are cited.

3 R. Jost, Helv. Phys, Acta 20, 256 (1947).

4 S. N. Biswas, T. Pradhan, and E. C. G. Sudarshan, University of Texas at Austin preprint (September 1970).

5 T.D. Lee and G. C. Wick, Nucl. Phys. B9, 209 (1969); B10, 1 (1969); T. D. Lee, CERN Report No. Th. 914 (unpublished). A. M. Gleeson and E.C.G. Sudarshan, Phys. Rev. D 1, 474 (1970).

6 E. C. G. Sudarshan, "Action at a Distance", Report to the Austin Symposium on the Past Decade in Particle Theory, April 1970; Fields and Quanla, Vol. II (in press). C. A. Nelson and E.C.G. Sudarshan, University of Texas at Austin, Preprint CPT-94, AEC-44 (March 1970); Austin preprint (April 1970).

7 The relativistic wave equations (2.3) and $(2.20)$ have the same differential equation structure as that of the Schrödinger equation and so the approach of Jost may be used. The definitions and notations employed here is that of R. G. Newton, Scattering Theory of Waves and Particles, (McGraw-Hill, New York, 1966).

8 G. S. Guralnik and C. R. Hagen, Phys. Rev. 130, 1259 (1963).

9 See A.Erdélyi (Ed.), Bateman Manuscript Project: Higher Trans cendental Funclions (McGraw Hill, New York, 1953) Vol. I, Chap. VI. Volumes in this series are hereafter referred to as HTFI, II, III.
10 HTFI-6-13-(6).

11 R. Jost and A. Pais, Phys. Rev. 82, 840 (1951).

1.2 E. C. G. Sudarshan, in Leclures in Theoretical Physics, Brandeis Summer Institule, 1961 (Benjamin, New York, 1962).

13 B. Friedman, Principles and Techniques of Applied Mathematics, (Wiley, New York, 1956).

14 The boundary conditions of $\varphi(k ; r)$ and $f_{ \pm}(k, r)$ at $r=0$ and $r \rightarrow \infty$, respectively, are the same as for a local potential.

15 An alternative procedure is to introduce a proper regular solution $\bar{\varphi}(k, r)=C(k) \varphi(k, r)$ and a proper regular Green's function $\overline{\mathfrak{g}}^{(+)}=C(k) \mathrm{S}^{(+)}$, i.e.,

$$
\begin{aligned}
& \lim _{r \rightarrow \infty} e^{\mp i k r} \bar{f}_{ \pm}(k, r)=C(k), \\
& \lim _{r \rightarrow 0} \bar{\varphi}(k, r)^{\prime}=C(k)
\end{aligned}
$$

and to work with these proper solutions as for a local theory, e.g., $\left.\psi^{(+)}(k, r)^{\prime}\right|_{r=0}=\left.k \varphi(k, r)^{\prime}\right|_{r=0} / \bar{f}_{+}(k, 0)$. It is permissible to modify the boundary conditions for $f_{+}(k, r)$ and $\varphi(k, r)$ as they are mathematical constructs; however, see the discussion in the text concerning the Wronskian. Note also C.S. Warke and R. K. Bhaduri, Nucl. Phys. A162, 289 (1971).

16 T. D. Lee, Phys. Rev. 95, 1329 (1954).

\title{
Electromagnetic Radiation in a Uniformly Moving, Homogeneous Medium
}

\author{
Günther Johannsen
}

Danish Engineering Academy, Electrical Department, The Technical University of Denmark, Aalborg, Denmark (Received 12 July 1971)

A new method of treating radiation problems in a uniformly moving, homogeneous medium is presented. A certain transformation technique in connection with the four-dimensional Green's function method makes it possible to elaborate the Green's functions of the governing differential equations in the rest system of the medium, whereas the final integrals determining the field may be calculated in the rest system of the source.

\section{INTRODUCTION}

In recent years several papers have been concerned with electromagnetic radiation in a uniformly moving medium with a more or less complicated structure. The usual approach is to formulate the governing differential equation in the observer system (rest system of the source) $K$ and then evaluate the solution for this equation. ${ }^{1-11}$

In two articles ${ }^{12,13}$ the author showed that advantage may be derived from the fact that the governing differential equation s are simplest in the rest frame $K^{\prime}$ of the medium, the medium being simple (i.e., 
homogeneous, isotropic, lossless and non dispersive) and conducting (but otherwise simple), respectively.

It is the purpose of the present paper to show that a certain transformation technique in connection with the four-dimensional Green's function method makes it possible to derive advantage from both the "simplicity" of the governing differential equation in $K^{\prime}$ and the "simplicity" of the source distribution in $K$; the structure of the medium may be rather complicated.

The basic idea of the method can be described in this way: The first step in the Green's function technique is to find a fundamental solution corresponding to a source which is represented by a four-dimensional $\delta$ function. Such a space-time impulse is bound to a single space-time point (world point). As far as we are concerned with a single world point, all inertial systems are equally suitable, and therefore, in the first step, the rest system of the medium should be used because the governing differential equations are simplest in this system.

The next step consists of a summation over the world points of the source distribution. This step is in principle independent of the first one, and, therefore, the most convenient inertial system should be used for the second step. Usually, the rest system of the source (if such a system exists, of course) is the most suitable one for the summation procedure.

Mathematically, the formalism provides the definition of a Green's tensor function which can be evaluated in an arbitrary inertial frame. (In $K^{\prime}$ this function may even be well known from the corresponding radiation problem, where the medium is at rest relatively to the source). This Green's function can be transformed to another arbitrary inertial frame (e.g., the rest frame $K$ of the source), where the final integrals which represent the field may be worked out.

The general formalism is elaborated for a homogeneous medium and then applied to some examples: An isotropic medium and a lossless, electrically, uniaxially, anisotropic medium are considered. The first case has been treated before by Besieris and Compton, ${ }^{9}$ Chen and Yen, 10 and the author, ${ }^{13}$ the second case by S.W. Lee and Lo. ${ }^{6}$ Case one includes a simple medium (Refs. 1, 5, 8,12). Application of the method will also be made to a dispersive but otherwise simple medium (ionized gas), a case which has been treated before by K.S.H. Lee and Papas. ${ }^{4}$

\section{FORMAL SOLUTION IN THE REST SYSTEM OF THE MEDIUM}

In this section all quantities refer to the system of inertia $K^{\prime}$ in which the medium is at rest. The transformation of the results into four-dimensional tensor language will be done in the next section. In preparation for this transformation we will use three-dimensional, Cartesian tensor notation (cf. Ref.14) in this section. Latin subscripts run from 1 to 4 ; Greek subscripts run from 1 to 3 . The coordinate $x_{4} \equiv i c t$, where $t$ is the time and $c$ the speed of light in vacuum. Repeated subscripts obey the summation convention, and commas in subscripts denote partial differentiation with respect to coordinates.

The constitutive equations are given by

$$
\begin{aligned}
& D_{\kappa}^{\prime}=\epsilon_{\kappa \lambda} E_{\lambda}^{\prime}, \\
& B_{\kappa}^{\prime}=\mu_{\kappa \lambda} H_{\lambda}^{\prime}, \\
& J_{\kappa}^{\prime \prime}=\sigma_{\kappa \lambda} E_{\lambda}^{\prime},
\end{aligned}
$$

where the dyadics $\epsilon_{\kappa \lambda}, \mu_{\kappa \lambda}$, and $\sigma_{\kappa \lambda}$ are assumed to be independent of the space-time coordinates $x_{r}^{\prime}$ (i.e., the medium is homogeneous). $J_{k}^{0}$ denotes the free current density, whereas the free charge density $\rho^{0 \prime}$ is assumed to be vanishing.

A vector potential $A_{\kappa}^{\prime}$ and a scalar potential $A_{4}^{\prime} \equiv$ $(i / c) \Phi^{\prime}$ can be introduced in the usual way. The field is given by

$$
\begin{aligned}
\mu_{\kappa \lambda} H_{\lambda}^{\prime} & =\epsilon_{\kappa \lambda_{\nu}} A_{\nu, \lambda}^{\prime}, \\
E_{\kappa}^{\prime} & =i c\left(A_{4, \kappa}^{\prime}-A_{\kappa, 4}^{\prime}\right),
\end{aligned}
$$

where $\epsilon_{\kappa \lambda \nu}$ is the three-dimensional permutation symbol, and the differential equations for the potentials can be written as

$$
\begin{gathered}
\epsilon_{\kappa \lambda \nu} \epsilon_{\rho \sigma \tau} \mu_{\nu \tau}^{-1} A_{\sigma, \lambda \rho}+c^{2} \epsilon_{\kappa \lambda}\left(A_{4,4 \lambda}^{\prime}-A_{\lambda, 44}^{\prime}\right) \\
-i c \sigma_{\kappa \lambda}\left(A_{4, \lambda}^{\prime}-A_{\lambda, 4}^{\prime}\right)=J_{\kappa}^{\prime}, \\
c^{2} \epsilon_{\lambda \nu}\left(A_{\nu, \lambda 4}^{\prime}-A_{4, \lambda \nu}^{\prime}\right)=J_{4}^{\prime} .
\end{gathered}
$$

$J_{\kappa}^{\prime}$ is the current density of the source and $J_{4}^{\prime} \equiv i c \rho^{\prime}$, where $\rho^{\prime}$ is the charge density of the source. Finally $\mu_{\nu \tau}^{-1} \mu_{\tau \lambda}=\delta_{\nu \lambda}$ which is the three-dimensional Kronecker symbol. [The matrices representing the constitutive dyadics in (1) are assumed to be regular.]

Introducing the definitions $n^{2} \equiv c^{2} \mu \epsilon$ and

$$
\begin{aligned}
\epsilon_{\kappa \lambda}^{\star} & \equiv \epsilon^{-1} \epsilon_{\kappa \lambda}-\delta_{\kappa \lambda}, \\
\left(\mu_{\kappa \nu}^{-1}\right)^{\star} & \equiv \mu \mu_{\kappa \lambda}^{-1}-\delta_{\kappa \lambda}, \\
\sigma_{\kappa \lambda}^{\star} & \equiv \sigma^{-1} \sigma_{\kappa \lambda}-\delta_{\kappa \lambda},
\end{aligned}
$$

where $\epsilon, \mu, \sigma$ are constants (different from zero), we may write the differential equations (3) in the form

$$
\begin{gathered}
\epsilon_{\kappa \lambda \nu} \epsilon_{\rho \sigma \tau}\left(\mu_{\nu}^{-}\right)^{\star} A_{\sigma, \rho \lambda}^{\prime}+n^{2}\left[\epsilon_{\kappa \lambda}^{\star}\left(A_{4, \lambda 4}^{\prime}-A_{\lambda, 44}^{\prime}\right)\right. \\
\left.-(i / c)(\sigma / \epsilon) \sigma_{\kappa \lambda}^{\star}\left(A_{4, \lambda}^{\prime}-A_{\kappa, 4}^{\prime}\right)\right]-A_{\kappa, \lambda \lambda}^{\prime}-n^{2} \\
\times\left[A_{\kappa, 44}^{\prime}-(i / c)(\sigma / \epsilon) A_{\kappa, 4}^{\prime}\right]=\mu J_{\kappa}^{\prime}, \\
\epsilon_{\lambda \nu}^{\star}\left(A_{\nu, 4 \lambda}^{\prime}-A_{4, \nu \lambda}^{\prime}\right)-A_{4, \lambda \lambda}^{\prime}-n^{2}\left[A_{4,44}^{\prime}-(i / c)(\sigma / \epsilon)\right. \\
\left.\times A_{4,4}^{\prime}\right]=\mu J_{4}^{\prime} / n^{2} .
\end{gathered}
$$

In (5) we also have applied the Gauge condition

$$
A_{\lambda, \lambda}^{\prime}+n^{2}\left[A_{4,4}^{\prime}-(i / c)(\sigma / \epsilon) A_{4}^{\prime}\right]=0
$$

in the usual way ${ }^{15}$ to the isotropic terms of the equations.

Applying fourfold space-time Fourier transforms [cf.(12), Sec. III] to the differential equations (5), we obtain the matrix equation

$$
\left(M_{r s}^{\prime}+V^{\prime} \delta_{r s}\right) a_{s}^{\prime}=s_{r}^{\prime},
$$


where

$$
\begin{aligned}
M_{\kappa \lambda}^{\prime} \equiv-\epsilon_{\kappa \sigma \nu} \epsilon_{\rho \lambda \tau}\left(\mu_{\nu \tau}^{-}\right)^{\star} k_{\rho}^{\prime} k_{\sigma}^{\prime} \\
\quad+n^{2}\left[k_{4}^{\prime} \epsilon_{\kappa \lambda}^{\star}-(i / c)(\sigma / \epsilon) k_{4}^{\prime} \sigma_{\kappa \lambda}^{\star}\right], \\
M_{\kappa 4}^{\prime} \equiv-n^{2}\left[k_{4}^{\prime} \epsilon_{\kappa \lambda}^{\star}-(i / c)(\sigma / \epsilon) \sigma_{\kappa \lambda}^{\star}\right] k_{\lambda}^{\prime}, \\
M_{4 k}^{\prime} \equiv-\epsilon_{\lambda k}^{\star} k_{\lambda}^{\prime} k_{4}^{\prime}, \\
M_{44}^{\prime} \equiv+\epsilon_{\kappa \lambda}^{\star} k_{\kappa}^{\prime} k_{\lambda}^{\prime}, \\
V^{\prime} \equiv k_{\lambda}^{\prime} k_{\lambda}^{\prime}+n^{2}\left[k_{4}^{\prime 2}-(1 / c)(\sigma / \epsilon) k_{4}^{\prime}\right],
\end{aligned}
$$

and

$$
s_{k}^{\prime} \equiv \mu\left(j_{\kappa}^{\prime}, j_{4}^{\prime} / n^{2}\right) \text {. }
$$

It is noticed that $M_{r s}^{\prime}$ vanishes when the medium is isotropic.

Defining

$$
g_{r s}^{\prime} \equiv\left(M_{r s}^{\prime}+V^{\prime} \delta_{r s}\right)^{-1},
$$

we finally get

$$
a_{r}^{\prime}=g_{r t}^{\prime} s_{t}^{\prime}
$$

which establishes the formal solution of the problem in the transformed domain.

\section{TRANSLATION INTO FOUR-DIMENSIONAL TENSOR FORMALISM}

Consider a tensor field $T \ldots\left(x_{r}\right)$ of a rbitrary order (we use four-dimensional tensors as in Ref.12) and a fourfold Fourier integral

$$
\begin{aligned}
T \ldots\left(x_{r}\right) & =\iint_{-\infty}^{\infty} \int_{-i \infty}^{i \infty} t \ldots\left(k_{r}\right) e^{i k_{s} x_{s}} d\left(k_{r}\right) \\
& =\int_{M_{4}} t \ldots\left(k_{r}\right) e^{i k_{s} x_{s}} d\left(k_{r}\right),
\end{aligned}
$$

where the integration is to be taken over the whole Minkowski 4-space $M_{4}$.

A proper Lorentz transformation can be applied to the integration variables $k_{r}$ as well as to the spacetime variables $x_{r}$. The inner tensor product $k_{r} x_{r}$ is invariant, and we still have to integrate over the whole space $M_{4}$ because a Lorentz transformation is a one-to-one mapping of $M_{4}$ on itself. Therefore, the Fourier amplitudes $t$... transform like a tensor if $T$... does, and vice versa (because the transformation matrix does not depend on $x_{r}$ ).

The four-current density $J_{r}$ transforms like a tensor (cf.Ref.16) and so does the four-potential $A_{r}$ (cf. below). Therefore, the Fourier amplitudes $j_{r}$ and $a_{r}$ are tensors of order one (4-vectors).

We make also use of the 4-vector

$$
S_{r} \equiv S_{r s} J_{s}, \quad S_{r s} \equiv \mu\left(\delta_{r s}+\kappa / n^{2} U_{s} U_{r}\right),
$$

where $\kappa \equiv\left(n^{2}-1\right) / c^{2}$ and $U_{r}$ is the 4-velocity, i.e., $U_{r}^{\prime}=(0,0,0, i c)$ and $S_{r}^{\prime}=\left(J_{0}^{\prime}, J_{4}^{\prime} / n^{2}\right)$. It is seen that the Fourier amplitudes of $S_{r}^{\prime}$ are given by (9).

Furthermore, we define a second-order tensor $g_{r s}$ by means of the components in $K^{\prime}$ as given by (10). The tensor character of Eq. (11) now ensures that

$$
a_{r}=g_{r t} s_{t}
$$

is valid in an arbitrary inertial frame, and, referring to the statements made in the beginning of this section, we are able to give a covariant, formal solution of the problem:

where

$$
A_{n}\left(x_{r}\right)=\frac{1}{(2 \pi)^{4}} \int_{M_{4}} G_{n s}\left(u_{r}\right) J_{s}\left(z_{r}\right) d\left(z_{r}\right),
$$

$$
G_{n s}\left(u_{r}\right) \equiv \int_{N_{4}} g_{n m}\left(k_{r}\right) S_{m s} e^{i k_{t}{ }^{u} t} d\left(k_{r}\right), \quad u_{t} \equiv x_{t}-z_{t} \text {. }
$$

For a nondispersive medium, $n$ (and therefore also $S_{r s}$ ) is independent of the variables $k_{r}$. In that case we use a modified definition of the Green's tensor function:

$$
A_{n}\left(x_{r}\right)=\frac{1}{(2 \pi)^{4}} \int_{M_{4}} G_{n s}\left(u_{r}\right) S_{s}\left(z_{r}\right) d\left(z_{r}\right),
$$

where

$$
G_{n s}\left(u_{r}\right) \equiv \int_{M_{4}} g_{n s}\left(k_{r}\right) e^{i k_{t} u_{t}} d\left(k_{r}\right)
$$

Since the Green's terisor function (16) is simplest in $K^{\prime}$ (which is a consequence of the fact that the governing differential equations are simplest in $K^{\prime}$ ), an evaluation of the integral in (16) should be sought (may be well known) in the rest frame of the medium (cf. the following sections).

In Minkowski's theory the field quantities $E_{\kappa}, B_{\kappa}, H_{\kappa}$, $D_{k}$ transform in a certain manner from one inertial frame to another, which is called the Lorentz transformation of the field. This can be expressed very briefly by saying that the field quantities constitute the components of two second-order field tensors $F_{r s}$ and $H_{r s}$ (consult Ref. 17 for the definition).

Define a 4-vector $A_{r}$ in such a way that the $A_{r}^{\prime}$ are identical with the potentials entering Eqs. (2), then the tensor equation

$$
F_{r s}=c\left(A_{s, r}-A_{r, s}\right)
$$

is valid because (17) is equivalent to (2) in the rest system $K^{\prime}$, which in turn shows that the primes may be omitted in (2b), whereas the spatial part of (17) is equivalent to the equation $B_{\kappa}=\epsilon_{\kappa \lambda \nu} A_{\nu, \lambda}$.

In order to express the field quantities $H_{\kappa}$ and $D_{\kappa}$ by means of the potential, this can only be done via the constitutive relations. Therefore, it might be convenient to have covariant forms of these relations to our disposal.

Following $\operatorname{Marx}^{18}$ in a slightly deviating way, we define

$$
\begin{aligned}
F_{r} & \equiv(1 / c) F_{r s} U_{s}, \\
\tilde{F}_{r} & \equiv\left(1 / i c^{2}\right) F^{\star}{ }_{r s} U_{s}, \\
K_{r} & \equiv\left(1 / c^{2}\right) H_{r s} U_{s}, \\
\tilde{K}_{r} & \equiv(1 / i c) H_{r s}^{\star} U_{s},
\end{aligned}
$$

where $F_{r s}^{\star}, H_{r s}^{\star}$ are dual tensors, i.e., $F_{r s}^{\star} \equiv(1 / 2 !)$ $\epsilon_{r s m n} F_{m n}$, say. It is readily seen that $F_{r}^{\prime}=\left(E_{\rho}^{\prime}, 0\right)$, $\widetilde{F}_{r}^{\prime}=\left(B_{\rho}^{\prime}, 0\right), K_{r}^{\prime}=\left(D_{\rho}^{\prime}, 0\right)$, and $\tilde{K}_{r}^{\prime}=\left(H_{\rho}^{\prime}, 0\right)$. 
By defining tensors $e_{r s}, m_{r s}$ by means of $e_{\rho 0}^{\prime} \equiv \epsilon_{\rho \sigma}$, $m_{\rho \sigma}^{\prime} \equiv \mu_{\rho \sigma}, \quad e_{4 \sigma}^{\prime} \equiv m_{4 \sigma}^{\prime} \equiv 0$ (the remaining components can be chosen arbitrarily), it follows from (1) that

$$
\begin{aligned}
& K_{r}=e_{r s} F_{s}, \\
& F_{r}=m_{r s} \tilde{K}_{s} .
\end{aligned}
$$

Using tensor relations inverse to (18) (expressing second-order field tensors in terms of first order field tensors), one can express $H_{r s}$ in terms of $F_{r s}$, say (cf. Ref. 18). Marx ${ }^{18}$ proposed that the components $e_{r 4}^{\prime}, m_{r 4}^{\prime}$ are vanishing. In order to get regular matrices, we choose $e_{\rho 4}^{\prime} \equiv m_{\rho 4}^{\prime} \equiv 0, \quad \epsilon_{44}^{\prime} \equiv \epsilon$, and $m_{44}^{\prime} \equiv \mu$. If wanted, even a covariant, four-dimensional formulation of the "wave equations" can be obtained from (5) by means of a simple translation technique. (The usual way is to introduce 4-potentials into the 4-tensor formulation of Maxwell-Minkowski equations, as was done by Viglin 7 and earlier by Jauch and Watson 19 for the isotropic case.) Defining $m_{r s}^{-1}$ such that $m_{r s}^{-1} m_{s t}$ $=\delta_{r t}$, we obviously have $\left(m_{\rho \sigma}^{-1}\right)^{\prime}=\mu_{0 \sigma}^{-1}, \quad\left(m_{44}^{-1}\right)^{\prime}=1 / \mu$. Furthermore, the definitions $e_{r s}^{\star} \equiv(1 / \epsilon) e_{r s}-\delta_{r s}$, $\left(m_{r s}^{-1}\right)^{\star} \equiv \mu\left(m_{r s}^{-1}\right)-\delta_{r s}$ imply that the spatial parts of these tensors in $K^{\prime}$ are given By (4) and that the remaining components in $K^{\prime}$ are equal to zero.

Consider the first term of Eq. (5a); we get

$$
\begin{aligned}
\epsilon_{\kappa \lambda \nu} \epsilon_{\rho \sigma \tau}\left(\mu_{\nu \tau}^{-1}\right)^{\star} A_{\sigma, \rho \lambda}^{\prime} & =\epsilon_{\kappa l n 4} \epsilon_{r s t 4}\left(m_{n t}^{-1}\right)^{\star} A_{s, r l}^{\prime} \\
& =-\left(1 / c^{2}\right) \epsilon_{\kappa l n u} \epsilon_{r s t \nu}\left(m_{n t}^{-1}\right)^{\star} A_{s, r l}^{\prime} U_{u}^{\prime} U_{v}^{\prime} .
\end{aligned}
$$

It is observed that the "fourth component" ( $\kappa$ is replaced by the integer 4 in the final expression) vanishes.

The second term in Eq. (5a) is translated in this way: $n^{2} \epsilon_{\kappa \lambda}^{\star}\left(A_{4, \lambda 4}^{\prime}-A_{\lambda, 44}^{\prime}\right)=-\frac{n^{2}}{c^{2}} e_{\kappa l}^{* \prime}\left(A_{r, l s}^{\prime}-A_{l, r s}^{\prime}\right) U_{r}^{\prime} U_{s}^{\prime}$.

Again, it is noticed that the fourth component of the final expression vanishes.

In order to translate the third term of (5a), we define tensors $s_{r s}$ and $s_{r s}^{*}$ by means of $s_{\rho \sigma}^{\prime}, \equiv \sigma_{\rho \sigma}, s_{4 \rho}^{\prime} \equiv s_{\rho 4}^{\prime} \equiv 0$, $s_{44}^{\prime} \equiv \sigma, s_{r s}^{\star} \equiv s_{r s} / \sigma-\delta_{r s}$. Since $s_{\rho \sigma}^{\star \prime}=\sigma_{\kappa \lambda}^{\star}[\mathrm{cf} .(4)]$, we conclude that

$-(i \sigma / c \epsilon) \sigma_{\kappa \lambda}^{\star}\left(A_{4, \lambda}^{\prime}-A_{\lambda, 4}^{\prime}\right)=-\left(\sigma / c^{2} \epsilon\right) s_{\kappa l}^{\star \prime}\left(A_{u l}^{\prime}-A_{l u}^{\prime}\right) U_{u}^{\prime}$

and that the fourth component of the final expression is zero.

The first term of (5b) is equal to the fourth component of $-\left(1 / c^{2}\right) e_{l n}^{\star \prime}\left(A_{n, r l}^{\prime}-A_{r, n l}^{\prime}\right) U_{r}^{\prime} U_{k}^{\prime}$, whereas the first three components are equal to zero.

It is not difficult to translate the remaining, isotropic terms of (5a) and (5b) into the four-dimensional form of the isotropic wave equation.

Omitting primes, we finally get a 4-tensor formulation of Eq.(5):

$$
\begin{gathered}
\left(1 / c^{2}\right)\left\{\left[\epsilon_{k l n u} \epsilon_{r s t}\left(m_{n t}^{-1}\right)^{\star} A_{s, r l}+n^{2} e_{k l}^{\star}\left(A_{u, l v}-A_{l, u v}\right)\right] U_{u} V_{v}\right. \\
\left.-(\sigma / \epsilon) s_{k l}^{\star}\left(A_{u, l}-A_{l, u}\right) U_{u}+e_{l n}^{\star}\left(A_{n, r l}-A_{r, n l}\right) U_{r} U_{k}\right\} \\
+A_{k, n n}-\left[\left(n^{2}-1\right) / c^{2}\right] A_{k, r s} U_{r} U_{s}=-S_{k} .
\end{gathered}
$$

Finally, we want to make some remarks on dispersive media. In $K^{\prime}$ the constitutive parameters of a dispersive medium are assumed to be functions of the frequency $\omega^{\prime}=c k_{4}^{\prime} / i$ corresponding to a Fourier component of the field.

Since the formalism provides a decomposition of the field into Fourier components in all inertial frames, we may define a "frequency" referring to an arbitrary inertial system $K$ :

$$
\omega \equiv c k_{4} / i \text {. }
$$

In order to get covariant forms for the functions which express the dependency of the constitutive parameters on $\omega^{\prime}$, we observe that

$$
\omega^{\prime}=-k_{r}^{\prime} U_{r}^{\prime}=-k_{r} U_{r}
$$

Clearly, (22) shows that in $K$ (different from $K^{\prime}$ ) the constitutive parameters depend not only on $k_{4}$ (i.e., $\omega)$ but also on the spatial part $k_{\rho}$ of the "propagation tensor" $k_{r}$.

\section{ISOTROPIC, NONDISPERSIVE MEDIUM}

For an isotropic, nondispersive medium it is seen from (4), (8), and (10) that $\epsilon_{k \lambda}^{\star}=0$, etc., $M_{r s}^{\prime}=0$, and $g_{r s}^{\prime}=\left(1 / V^{\prime}\right) \delta_{r s}$. Defining a Green's function $G^{\prime}\left(u_{t}^{\prime}\right)$ such that $G_{r s}^{\prime}\left(u_{t}^{\prime}\right)=G^{\prime}\left(u_{t}^{\prime}\right) \delta_{r s}$ we find that this function is given by the integral [cf.(16a)]

$$
G^{\prime}\left(u_{r}^{\prime}\right)=\int_{M_{4}} \frac{1}{V^{\prime}\left(k_{r}^{\prime}\right)} e^{i k_{t}^{\prime} u_{t}^{\prime}} d\left(k_{r}^{\prime}\right),
$$

where $V^{\prime}\left(k_{r}^{\prime}\right)$ is given by (8e).

The substitution $k_{4}^{\prime}+i l_{4}^{\prime} \rightarrow k_{4}^{\prime}, \quad l_{4}^{\prime} \equiv(i / 2 c)(\sigma / \epsilon)$ leads to

$$
\begin{aligned}
G^{\prime}\left(u_{r}^{\prime}\right)= & e^{l_{4}^{\prime} u_{4}^{\prime}} \iiint_{-\infty}^{\infty} \int_{\alpha-i \infty}^{\alpha+i_{\infty}} \\
& \times \frac{e^{i k_{t^{\prime} u_{t}^{\prime}}}}{k_{\lambda}^{\prime} k_{\lambda}^{\prime}+n^{2}\left(k_{4}^{\prime 2}+l_{4}^{\prime 2}\right)} d\left(k_{r}^{\prime}\right), \alpha \equiv-\left|l_{4}^{\prime}\right| .
\end{aligned}
$$

The integral in (21) is the Fourier integral of the time-dependent Green's function of the Klein-Gordon differential equation. Since $G$ is a tensor of zero order (Lorentz invariant) the Lorentz transformation of this well-known function 20 to an arbitrary inertial frame can be achieved by translating the dependency of this function on the variables $u_{r}$ into covariant tensor language, as was done by the author in Ref.13. This is easier than evaluating the integrals in the covariant edition of (24) which is given by

$G\left(u_{r}\right)=e^{l_{t}{ }^{u} t} \int_{M_{4}} \frac{e^{i k_{t} u_{t}}}{k_{t} k_{t}-\kappa\left(k_{t} U_{t}\right)^{2}-\left[(n / c) l_{t} U_{t}\right]^{2}} d\left(k_{r}\right)$,

where we have defined a 4-vector $l_{r}$ such that $l_{r}^{\prime}=$ $(0,0,0,(i / 2 c)(\sigma / \epsilon))$.

The "difficult" way of evaluating (25) was used by Chen and Yen ${ }^{10}$ and, for the lossless case, by Compton. ${ }^{5}$ Earlier K.S.H. Lee and Papas ${ }^{1}$ evaluated the se integrals for a time-harmonic source in a simple medium. [Harmonic time dependency implies that the $k_{4}$-integration in (25) "disappears". This is readily seen from (15a) in connection with (25): 
Assuming that $S_{s}$ in (15a) is time-harmonic, the $z_{4}-$ integration introduces a time-dependent $\delta$ function in (24)]

\section{IONIZED GAS}

Consider a medium which is dispersive but otherwise simple (i.e., isotropic and lossless). Furthermore, assume that $\mu=\mu_{0}$ and

$$
\epsilon\left(\omega^{\prime}\right)=\epsilon\left(-k_{r} U_{r}\right)=\epsilon_{0}\left[1+\left(c k_{(p)} / k_{r} U_{r}\right)^{2}\right],
$$

where $\mu_{0}, \epsilon_{0}$ are the permeability and the dielectric constant of vacuum, $k_{(p)} \equiv i \omega_{(p)} / c$, and $\omega_{(p)}$ is a real number.

Following Ref.4, we wish to evaluate the Green's function for the unbounded space in the form of a onedimensional integral, but our approach is different from that in Ref.4: A well-known result referring to the rest frame $K^{\prime}$ of the medium is transformed to $K$. In the case which is under consideration Eq. (16) reduces to

$$
G_{n s}^{\prime}\left(u_{t}^{\prime}\right)=\mu_{0} \int_{M_{4}} \frac{\delta_{n s}-\kappa / n^{2} U_{n}^{\prime} U_{s}^{\prime}}{k_{\lambda}^{\prime} k_{\lambda}^{\prime}+n^{2} k_{4}^{\prime 2}} e^{i k_{t}^{\prime} u_{t}^{\prime}} d\left(k_{r}^{\prime}\right),
$$

where we have made use of (13).

It is well known from the corresponding radiation problem in a nonmoving and nondispersive medium that the threefold space integral in (27) can be reduced to a single integral (of course, this result can be obtained by exactly the same steps which are used in Ref. 4 but with the simplification that $v$ is zero).

We have

$$
\begin{aligned}
G_{n s}^{\prime}\left(u_{t}^{\prime}\right)= & i \pi^{2} \mu_{0} \int_{-i \infty}^{i \infty} \int_{\infty}^{\infty} \\
& +\left[\delta_{n s}+\left(\kappa / n^{2}\right) U_{n}^{\prime} U_{s}^{\prime}\right] H_{0}^{1}\left[\rho \sqrt{-\left(k_{3}^{\prime 2}+n^{2} k_{4}^{\prime 2}\right)}\right] \\
& \times e^{i\left(k_{3}^{\prime} u_{3}^{\prime}+k_{4}^{\prime} u_{4}^{\prime}\right)} d k_{3}^{\prime} d k_{4}^{\prime},
\end{aligned}
$$

where $\rho^{\prime} \equiv\left(u_{1}^{\prime 2}+u_{2}^{\prime 2}\right)$ and $H_{\delta}^{1}$ is the Hankel function of zero order and first kind.

By means of (26), the argument of the Hankel function can be written as $\rho^{\prime}\left[k^{2}(p)-\left(k_{3}^{\prime 2}+k_{4}^{\prime 2}\right)\right]^{1 / 2}$.

As in Ref. 4 we choose (without loss of generality) the velocity of $K$ relative to $K^{\prime}$ to be directed along the $x_{3}\left(x_{3}^{\prime}\right)$ axis. This implies that the first and the second component of a 4-vector are uneffected by the Lorentz transformation so that $\rho^{\prime}=\rho$. Furthermore, $k_{3}^{\prime 2}+$ $k_{4}^{\prime 2}=k_{3}^{2}+k_{4}^{2}$ and $k_{3}^{\prime} u_{3}^{\prime}+k_{4}^{\prime} u_{4}^{\prime}=k_{3} u_{3}+k_{4} u_{4}$ (because these expressions are tensors of zero order, i.e., invariants).

The transformation of (27) to an arbitrary inertial frame $K$ is now almost trivial. Eliminating $\kappa / n^{2}$ by means of (26), we obtain

$$
\begin{aligned}
G_{n s}\left(u_{t}\right)= & i \pi^{2} \mu_{0} \int_{-i \infty-\infty}^{i \infty} \int_{\infty}^{\infty}\left(\delta_{n s}+\frac{k{ }_{p)} U_{n} U_{s}}{c^{2} k_{(p)}^{2}+\left(k_{r} U_{r}\right)^{2}}\right) \\
& \times H_{0}^{1}\left[\rho \sqrt{k_{(p)}^{2}-\left(k_{3}^{2}+k_{4}^{2}\right)}\right] e^{i\left(k_{3} u_{3}+k_{4} u_{4}\right)} d k_{3} d k_{4} .
\end{aligned}
$$

The first term of this expression (containing $\delta_{n s}$ ) may be integrated with respect to $k_{3}$, leading to the result of Ref.4.
On the other hand, it can be done in this way: Since $k_{\lambda}^{\prime} k_{\lambda}^{\prime}+n^{2} k_{4}^{\prime 2}=k_{r}^{\prime} k_{r}^{\prime}-k{ }_{(p)}^{2}=k_{r} k_{r}-k 2_{(p)}^{2}$, the first term of (27) can be written as

$$
\mu_{0} \delta_{n s} \int_{-i \infty}^{i \infty} e^{i k_{4} u_{4}} \iint_{-\infty}^{\infty} \int_{\frac{1}{k^{2}}} \frac{e^{i u_{\tau}}}{k_{\nu} k_{\nu}-\left(k_{(p)}^{2}-k_{4}^{2}\right)} d\left(k_{\rho}\right) d k_{4} .
$$

This expression is covariant (!), and the spatial integral of this term is well known (from the simple wave equation) and is equal to $\exp \left[\operatorname{ir}\left(k_{(p)}^{2}-k_{4}^{2}\right)^{1 / 2}\right] / 4 \pi r$ in agreement with Ref.4.21

\section{LOSSLESS, ELECTRICALLY, UNIAXIALLY ANISOTROPIC MEDIUM}

Consider a medium for which $\sigma, \sigma_{\kappa \lambda}^{\star}$, and $\left(\mu_{\kappa \lambda}^{-1}\right)$ are vanishing and

$$
\epsilon_{\kappa \lambda}^{\star}=\left(\begin{array}{lll}
0 & 0 & 0 \\
0 & 0 & 0 \\
0 & 0 & \tau-1
\end{array}\right)
$$

where $\tau$ is a given positive constant [cf. (4)].

$M_{k l}$ as given by (8) reduces considerably, and $g_{r s}^{\prime}$ is readily found from (10). We get

$$
g_{r s}^{\prime}\left(k_{t}^{\prime}\right)=\left(\begin{array}{cccc}
1 / V^{\prime} & & 0 & 0 \\
0 & 1 / V^{\prime} & 0 & 0 \\
0 & 0 & \frac{X^{\prime}}{V^{\prime} Z^{\prime}} \frac{(\tau-1) n^{2} k_{3}^{\prime} k_{4}^{\prime}}{V^{\prime} Z^{\prime}} \\
0 & 0 & \frac{(\tau-1) k_{3}^{\prime} k_{4}^{\prime}}{V^{\prime} Z^{\prime}} & \frac{Y^{\prime}}{V^{\prime} Z^{\prime}}
\end{array}\right),
$$

where $V^{\prime}$ is given by (8e) and

$$
\begin{aligned}
& X^{\prime} \equiv k_{1}^{\prime 2}+k_{2}^{\prime 2}+\tau k_{3}^{\prime 2}+\left(n k_{4}^{\prime}\right)^{2}, \\
& Y^{\prime} \equiv k_{1}^{\prime 2}+k_{2}^{\prime 2}+k_{3}^{\prime 2}+\tau\left(n k_{4}^{\prime}\right)^{2}, \\
& Z^{\prime} \equiv k_{1}^{\prime 2}+k_{2}^{\prime 2}+\tau k_{3}^{\prime 2}+\tau\left(n k_{4}^{\prime}\right)^{2} .
\end{aligned}
$$

If we restrict the discussion to inertial frames moving on the axis of symmetry (i.e., the $x_{3}^{\prime}$ direction) it is consistent (i.e., independent of the inertial frame) to define two 4-vectors $s_{r}^{o}$ and $s_{r}^{e}$ in such a way that $s_{r}=s_{r}^{o}+s_{r}^{e}$ and $s_{3}^{o}=s_{4}^{o}=s_{1}^{e}=s_{2}^{e}=0$.

Case I: Consider a source for which $s_{r}^{e}=0$, e.g., a longitudinally orientated current loop (magnetic dipole). Obviously, $s_{1}=s_{1}^{\prime}, s_{2}=s_{2}^{\prime}$, and it is immediately seen from (31) that the only mode which is excited is identical with the isotropic case. This mode is called the ordinary or the first mode (cf. Ref.11).

Case II: Next, consider a source for which $s_{r}^{o}=0$, e.g., a longitudinally orientated, electrical dipole. Using the continuity equation $s_{3}^{\prime} k_{3}^{\prime}+s_{4}^{\prime}{ }^{2} k_{4}^{\prime}=0$, we derive, by means of (14) and (31), the equation

$$
g_{r t}\left(k_{n}\right) s_{t}=g^{e}\left(k_{n}\right) \delta_{r t} s_{t}, \quad g e\left(k_{n}\right) \equiv 1 / Z\left(k_{n}\right) .
$$

Obviously, only one mode is excited. The mode is of a 
different form than in Case I and is called the extraordinary or second mode (cf. Ref.11).

It is not difficult to find the corresponding extraordinary Green's function $G e$ from the ordinary Green's function $G^{\circ} \equiv G$ as given by (23), (24) (with $\sigma=0$ ).

The connection between the Green's functions is given by

$$
G e\left(u_{r}\right)=\tau^{-1} G^{o}\left(u_{1}, u_{2}, u_{3} / \sqrt{\tau}, u_{4} / \sqrt{\tau}\right) .
$$

In $K^{\prime},(34)$ is verified just by inspection of the corresponding fourfold Fourier integrals, and it is easily seen that the relation is covariant under those special Lorentz transformations which we are dealing with in this section.

$G\left(u_{r}\right)$ may be found in Ref.13. Applying the extraordinary Green's function $G e\left(u_{r}\right)$ to the case of a timeharmonic source, we readily derive the extraordinary Green's function as given by Lee and Lo. ${ }^{6}$

Case III: Finally, consider an arbitrary source distribution. From the continuity equation $s_{0}^{\prime} k^{\prime}+$ $n^{2} s_{4}^{\prime} k_{4}^{\prime}=0$ in connection with (14) and (31), we get

$$
a_{r}^{\prime}=\tilde{g}_{r t}^{\prime} s_{t}^{\prime},
$$

where we have defined a tensor $\tilde{g}_{r t}$ by means of the components in $K^{\prime}$ :

$\tilde{g_{r t}^{\prime}} \equiv\left(\begin{array}{cccc}1 / V^{\prime} & 0 & 0 & 0 \\ 0 & 1 / V^{\prime} & 0 & 0 \\ 0 & 0 & 1 / Z^{\prime} & 0 \\ 0 & 0 & 0 & 1 / Z^{\prime}\end{array}\right)$

$$
+\frac{1-\tau}{V^{\prime} Z^{\prime}}\left(\begin{array}{cccc}
0 & 0 & 0 & 0 \\
0 & 0 & 0 & 0 \\
k_{1}^{\prime} k_{3}^{\prime} & k_{2}^{\prime} k_{3}^{\prime} & 0 & 0 \\
k_{1}^{\prime} k_{4}^{\prime} & k_{2}^{\prime} k_{4}^{\prime} & 0 & 0
\end{array}\right)
$$

The contribution to the Green's function $\tilde{G}_{r t}^{\prime}$ corresponding to the first matrix term in (36) can immediately be obtained from Cases I and II. As to the second matrix term, the evaluation of the Fourier integral is more complicated. But, if $\tilde{G}_{r t}^{\prime}$ can be found in $K^{\prime}$, the transformation to another inertial system $K$ is simple. In fact, the second matrix in (27) is not affected at all by Lorentz transformations between inertial frames moving on the axis of symmetry. (Of course, the way in which the matrix depends on $k_{r}$ is affected.)

Though there is no need for working in $K$ at all (as was done in Ref.6), it is easily verified (in $K^{\prime}$ ) that

$$
\begin{aligned}
& V=k_{r} k_{r}+\left(1-n^{2}\right)\left(k_{r} U_{r} / c\right)^{2}, \\
& Z=k_{r} k_{r}+(\tau-1)\left(k_{r} T_{r}\right)^{2}+\left(1-\tau n^{2}\right)\left(k_{r} U_{r} / c\right)^{2},
\end{aligned}
$$

where we have defined a 4 -vector $T_{r}$ such that $T_{r}^{\prime}=$ $(0,0,1,0)$.

If the frequency $\omega$ is introduced by means of (21), the equations $V=0$ and $Z=0$ represent surfaces called the ordinary and extraordinary dispersion surface, respectively. These surfaces are of fundamental significance when asymptotic expressions of the Fourier integrals are to be evaluated (as was done in Ref.6), but this can more easily be done in $K^{\prime}$ (cf. Refs. 22, 23), whereupon the result can be translated to $K$ as outlined here.
1 K. S. H. Lee and C. H. Papas, J. Math. Phys. 5, 1668 (1964)

2 C.T.Tai, Trans. IEEE Antennas Propagation 13,322 (1965).

3 R. T. Compton, Jr. and C.T. Tai, Trans. IEEE Antennas Propagation 13,574 (1965).

4 K. S. H. Lee and C. H. Papas, Trans. IEE E Antennas Propagation 13, 799 (1965)

5 R. T. Compton, J. Math. Phys. 7, 2145 (1966).

6 S.W. Lee and Y.T. Lo, Radio Sci. 1, 313 (1966).

7 A.S. Viglin, Zh. Eksp. Teor. Fiz. 50, 85 (1966) [Sov. Phys. JETP 23, 59 (1966)]

8 C. T. Tai, J. Math. Phys. 8, 646 (1967).

9 I. M. Besieris and R. T. Compton, Jr., J. Math. Phys. 8, 2445 (1967).

10 K. C. Chen and J. L. Yen, J. Math. Phys. 9, 2081 (1968)

11 I. M. Besieris, J. Math. Phys. 10, 1156 (1969).

12 Günther Johannsen, J. Math. Phys.11, 3251 (1970).

13 Günther Johann sen, J. Math. Phys.12,1349 (1971)

14 J.L. Synge and A. Schild, Tensor Calculus (University of Toronto Press, Toronto, 1966).
15 D. S. Jones, The Theory of Electromagnetism (Macmillan, New York, 1964), pp. 14-15.

16 C. Moller, The Theory of Relativity (Oxford U.P., London, 1952), pp. 196-98.

17 A. Sommerfeld, Eleclrodynamics (Academic, New York, 1952), pp. 216-17.

18 G. Marx, Acta Phys. Acad. Sci.Hung. 3, 75 (1953).

19 J. M. Jauch and K. M. Watson, Phys. Rev. 74, 951 (1948).

20 P.M. Porse and H. Feshback, Methods of Theoretical Physics (McGraw-Hill, New York) Vol. I.,p. 856.

21 Attention is drawn to the fact that in $K^{\prime}$ Eq. (26) (i.e., $\epsilon / \epsilon_{0}=1-$ $\left.\omega_{(p)}^{2} / \omega^{\prime 2}\right)$ is assumed to hold for all $\omega^{\prime}$. This is true even if we assume harmonic time dependency in $K$ (as was done in Ref.4), because a spatial Fourier transformation in $K$ does not correspond to a pure spatial Fourier transformation in $K^{\prime}$.

22 G. A. Deschamps and O.Kesler, Trans. IEEE Antennas Propagation 11, 783 (1964).

23 M. J. Lighthill, Phil. Trans. Roy. Soc. London, Ser. A, 252, 397 (1960). 